\title{
Confiabilidade de medidas de arquitetura muscular do tríceps sural por ultrassonografia de imagem
}

\section{Reliability of triceps surae muscle architecture measurements obtained with sonographic imaging}

Natália Santos da Fonseca Martins ${ }^{1,2}$

Carolina Carneiro Peixinho ${ }^{1,2}$

Liliam Fernandes de Oliveira

Resumo - Há, atualmente, na literatura, poucas pesquisas de confiabilidade e validação da quantificação de parâmetros de arquitetura muscular por ultrassonografia (US), relatando informações importantes como o erro típico da medida (ETM) e o coeficiente de correlação intraclasse (ICC). O objetivo do estudo foi aplicar a técnica de US para quantificar as medidas de comprimento da fibra $(\mathrm{CF})$ e ângulo de penação $(\mathrm{AP})$ dos músculos gastrocnêmios e determinar a confiabilidade e a reprodutibilidade dessas medidas. Duas imagens de cada gastrocnêmio de 19 indivíduos jovens e saudáveis foram adquiridas com um equipamento de ultrassom modo- $\mathrm{B}(7,5 \mathrm{MHz})$, em duas visitas. $\mathrm{CF}$ e AP foram medidos cinco vezes nas duas imagens de cada indivíduo, totalizando vinte medidas de cada parâmetro para cada participante. A confiabilidade do total das 760 medidas foi determinada através do coeficiente de variação (CV), ICC e ETM. O CV do CF variou de 1,67 a $4,44 \%$ e do AP de 3,8 a $11,62 \%$. Todos os ICC se encontram no intervalo de grande confiabilidade, com exceção do valor referente à medida interdias do AP do gastrocnêmio medial $(0,67)$. Os dois valores mais altos de $\operatorname{ETM}\left(3,1^{\circ}\right.$ e $\left.3,7^{\circ}\right)$ correspondem aos dois valores inferiores do ICC $(0,67$ e 0,83$)$, referentes às medidas repetidas interdias dos gastrocnêmios. As medidas de $\mathrm{CF}$ dos gastrocnêmios medial e lateral apresentaram ETM de 2,17mm e 2,25mm, respectivamente. Os resultados obtidos demonstraram que a metodologia utilizada para medição de CF e AP realizada em imagens de ultrassom apresentou alta confiabilidade.

Palavras-chave: Arquitetura muscular; Confiabilidade; Ultrassonografia.

Abstract - There are few studies in the literature concerning the reliability and validity of the quantification of muscle architecture parameters using ultrasound that also report important information such as typical error of measurement (TEM) and intraclass correlation coefficient (ICC). The aim of this study was to use ultrasound imaging to quantify fiber length (FL) and pennation angle (PA) of the gastrocnemius muscle and to determine the reliability and reproducibility of these measurements. Two images of each gastrocnemius of 19 young healthy subjects were acquired using ultrasound equipment $(7.5 \mathrm{MHz})$, in two visits. FL and $P A$ were measured five times on each image, for a total of twenty measurements of each parameter. The overall reliability of the 760 measurements was determined through coefficients of variation (CV), ICC and TEM. The CV of FL ranged from 1.67 to $4.44 \%$ and from 3.8 to $11.62 \%$ for the PA. All ICC were within the high reliability range, with the exception of the between-visits PA value of the medial gastrocnemius (0.67). The two highest TEM values $\left(3.1^{\circ}\right.$ and $\left.3.7^{\circ}\right)$ correspond to the two lowest ICC values ( 0.67 and 0.83$)$, referring to repeated between-visit measurements of the medial and lateral gastrocnemius. FL measurements of the medial and lateral gastrocnemius had a TEM of 2.17 and 2.25 respectively. The results showed that ultrasound-based measurement of FL and PA exhibits high reliability.

Key words: Muscular architecture; Reliability; Ultrasound.
1 Universidade Federal do Rio de Janeiro. Laboratório de Biomecânica Muscular. Escola de Educação Física e Desportos. Rio de Janeiro, RJ. Brasil.

2 Universidade Federal do Rio de Janeiro. Laboratório de Ultrassom. Programa de Engenharia Biomédica. Rio de Janeiro, RJ. Brasil.

Recebido em 01/08/11 Revisado em 04/11/11 Aprovado em 07/11/11 


\section{INTRODUÇÃOO}

Medidas de parâmetros de arquitetura muscular têm sido cada vez mais utilizadas em estudos das propriedades contráteis do músculo tais como área de secção transversa anatômica e fisiológica, volume muscular, comprimento da fibra $(\mathrm{CF})$, espessura muscular e ângulo de penação $(\mathrm{AP})^{1-3}$. A arquitetura muscular sofre alterações devido a estímulos externos, condições patológicas ou estado fisiológico do indivíduo ${ }^{1,4}$. A plasticidade dos parâmetros biomecânicos de arquitetura muscular como o comprimento da fibra (CF) e o ângulo de penação (AP) tem sido analisada em diferentes condições de ativação, de uso aumentado ou reduzido ou em resposta aos diferentes programas de reabilitação e treinamento ${ }^{5-7}$. Inicialmente, valores de referência para arquitetura muscular eram extraídos de cadáveres, mas além do CF de um músculo em repouso ser menor que a fibra de um cadáver ${ }^{8}$, alterações fisiológicas do estado de rigor mortis e o processo de fixação dos tecidos comprometem a confiabilidade destes dados ${ }^{9}$.

Avanços nas técnicas de imagem, como a ultrassonografia (US), tornaram possível o estudo da arquitetura do músculo humano em tempo real e in vivo, de forma não-invasiva. Embora a ressonância magnética (RM) seja a técnica padrão ouro para a medição direta in vivo, é onerosa e demanda maior tempo de exame. A técnica de US, ao contrário, pode determinar aspectos da morfologia muscular, como área, comprimento e ângulo de penação do fascículo de modo objetivo e com baixo custo ${ }^{4}$, obtendo imagens de alta resolução de estruturas de diferentes tamanhos e profundidades, em tempo real, inclusive, em movimento ${ }^{5-7,9,10}$.

O CF e o AP são importantes parâmetros de arquitetura muscular e afetam as características funcionais de um músculo e a capacidade de produção da força ${ }^{10-12}$. O AP, definido como o ângulo de inserção das fibras musculares na aponeurose, relaciona-se à quantidade de tecido contrátil por unidade de área muscular. Alguns estudos mostram que em músculos hipertrofiados o AP encontra-se significativamente aumentado ${ }^{10}$, enquanto outros associam músculos com um maior $\mathrm{CF}$ a uma maior velocidade de contração ${ }^{12}$. Recentemente, foram constatados valores maiores de CF e menores de AP nos músculos gastrocnêmios (medial e lateral) e vasto lateral de corredores velocistas quando comparados a fundistas ${ }^{13}$. Muraoka et $\mathrm{l}^{14}$ apóiam a ideia de que um maior comprimento das fibras musculares e um maior número de sarcômeros em série estão associados à velocidade de contração. Alguns autores verificaram a relação entre o comprimento dos fascículos e ângulo articular do tornozelo, e a correlação desses com outros parâmetros arquitetônicos ${ }^{5,7,12}$.

Contudo, há poucas pesquisas de confiabilidade e validação da quantificação destes parâmetros ${ }^{5,6,15}$ através da US especialmente que relatem informações como o erro típico da medida (ETM) e o coeficiente de correlação intraclasse (ICC) que, quando não considerados, podem mascarar as diferenças existentes e dificuldades ocorridas devido ao protocolo de medição adotado (interavaliador, interdias, interimagens). A confiabilidade 
de uma medida pode ser ameaçada pelo grau de treinamento do avaliador e devido a maiores níveis de adiposidade subjacente ao local anatômico de realização das medidas, podendo prejudicar a identificação da interface músculo-tecido adiposo e consequentemente, a qualidade da medida ${ }^{16}$. Algumas das principais limitações nas análises residem na identificação pontual das interfaces teciduais (adiposo-muscular e muscular-ósseo) e na seleção de regiões de interesse, podendo induzir a erros significativos. A determinação do erro de medida relacionado ao protocolo de análise da imagem é fundamental para avaliação da função muscular, da estimativa da força e das adaptações do tecido em condições de uso aumentado ou desuso.

As técnicas de aquisição de imagens e de quantificação dos parâmetros variam entre os estudos, e a maioria não realiza ou relata resultados de análises de confiabilidade, dificultando a comparação de resultados e indicando a necessidade de adoção de uma metodologia reprodutível comum para pesquisas aplicadas.

O presente estudo avaliou a confiabilidade e variabilidade das medidas de CF e AP do gastrocnêmio lateral (GL) e do gastrocnêmio medial (GM), para análise da reprodutibilidade da técnica de US, quantificando o ETM e o ICC associados a estas medidas.

\section{PROCEDIMENTOS METODOLÓGICOS}

Participaram do estudo 19 voluntários (13 mulheres: $22,1 \pm 2,5$ anos $60,4 \pm 8,2 \mathrm{~kg}$ e $162 \pm 2 \mathrm{~cm}$ e 6 homens $23,3 \pm 7,2$ anos $72,7 \pm 7,8 \mathrm{~kg}$ e $169 \pm 2 \mathrm{~cm}$ ), fisicamente ativos, não participantes de um programa de treinamento regular e sem histórico de lesões ósteomioarticulares de membros inferiores. Os indivíduos assinaram um termo de consentimento livre e esclarecido antes da realização das medidas. O protocolo experimental foi aprovado pelo CEP da Instituição (número 03107).

Para aquisição das imagens, foi utilizado um aparelho de ultrassom (modelo EUB-405, Hitachi Medical Corporation, Tóquio, Japão), com um transdutor linear de $80 \mathrm{~mm}$ (frequência fundamental de 7,5MHz, campo de imagem de 65,0 x 80,3mm - largura x profundidade). O transdutor de tamanho relativamente grande $(80 \mathrm{~mm})$ quando comparado aos de outros estudos permitia um campo de imagem de $65,0 \mathrm{~mm}$ e, consequentemente, a medição do comprimento da fibra em uma única imagem sem necessidade de extrapolações. Foi utilizado, também, um gel (Ultrex-gel, Farmativa Indústria e Comércio Ltda, Rio de Janeiro, RJ, Brasil) para o acoplamento acústico e para evitar a depressão da superfície da pele. Para capturar as imagens do ultrassom, foi utilizada uma filmadora handcam Sony (AC-L25A, $50 \mathrm{a} 60 \mathrm{~Hz}$ ) e para a análise das medidas, o software ImageJ (National Institute of Health,

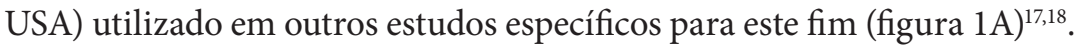

A aquisição das imagens do GM e do GL da perna direita foi feita pelo mesmo avaliador em duas visitas, com mínimo de 48 horas de intervalo, para verificação da confiabilidade de estabilidade ${ }^{19}$. Com o indivíduo em pé, marcava-se o local correspondente a $30 \%$ proximal do comprimento da perna (distância entre o maléolo medial e a interlinha articular do joelho), 
conforme protocolo de Miyatani et al $^{3}$. Em decúbito ventral, era identificada a junção dos gastrocnêmios medial e lateral e suas bordas para localização da linha média dos músculos. Em seguida, o transdutor era posicionado longitudinalmente em relação ao segmento na região marcada na perna onde eram escolhidas as imagens de melhor visualização do CF e do AP para cada músculo. Gravou-se uma imagem de cada músculo nos dois dias de visita para pós-processamento. Cada imagem (dia 1 e dia 2) foi analisada pelo mesmo avaliador cinco vezes através do software supracitado, totalizando dez medidas para cada parâmetro (CF e AP) e 20 medidas por músculo (GM e GL), contabilizando 760 medidas, 380 para cada parâmetro, em 19 indivíduos. Para medir o CF, selecionava-se na imagem a fibra de melhor resolução e com ferramenta do software para medida do comprimento de segmentos de reta eram determinados dois pontos cuja ligação correspondia à distância percorrida pela fibra da aponeurose superficial à profunda (Figura 1B). Para o AP, também com ferramenta do software, quantificava-se o ângulo formado por duas retas traçadas sobre a aponeurose profunda do músculo e sobre a fibra selecionada (Figura 1C), através da marcação de 3 pontos (um em cada reta e um na interseção entre as mesmas).

A análise estatística foi realizada no software Statistica 7.0 (Statsoft, Inc. Tulsa (OK) USA). A distribuição normal das medidas foi avaliada pelo teste Kolmogorov-Smirnov. Para verificar as diferenças das medidas dos dias e das cinco medições em cada imagem, aplicou-se o teste paramétrico ANOVA one-way para medidas repetidas em resposta a variáveis dependentes, com correção de Bonferroni. Verificou-se a confiabilidade e variabilidade das medidas pelo coeficiente de variação (CV), do ICC e para a estimativa de erro, calculou-se o erro ETM, sugerido por Hopkins ${ }^{20}$. O limite de significância estatística foi estabelecido em $\mathrm{p}<0,05$.

(A)

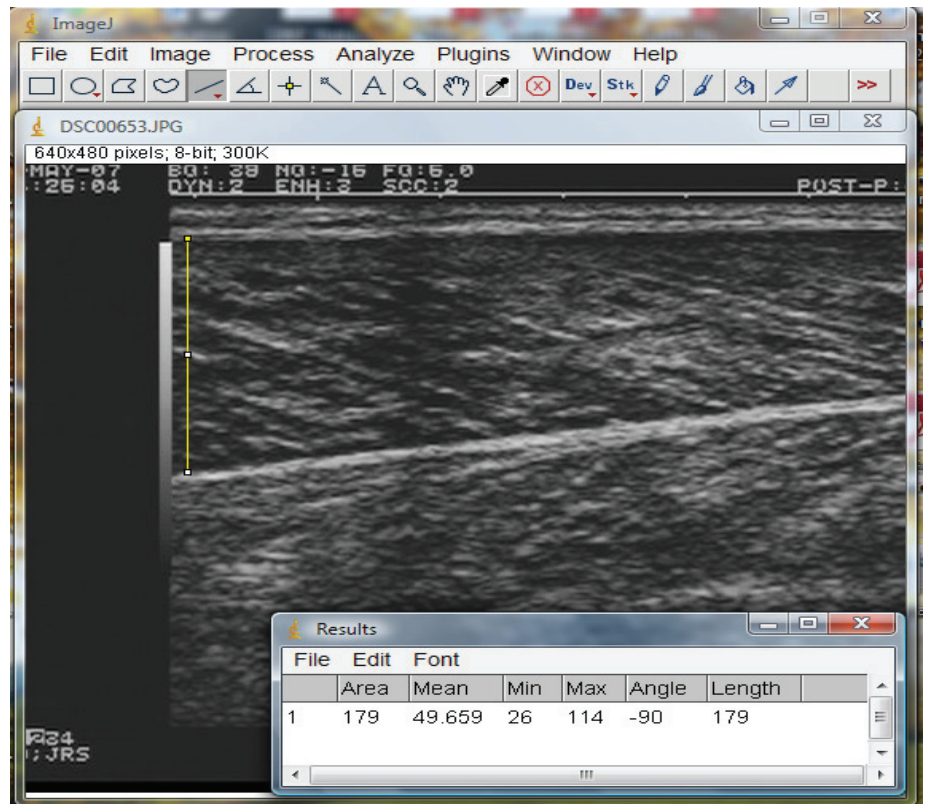

(B)

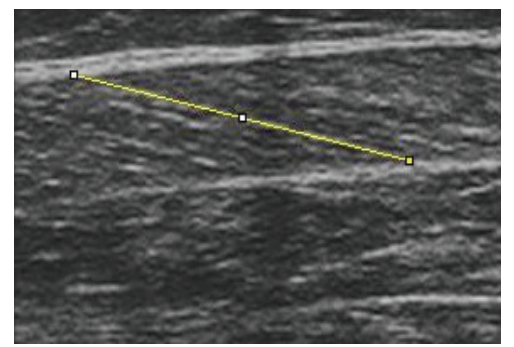

(C)

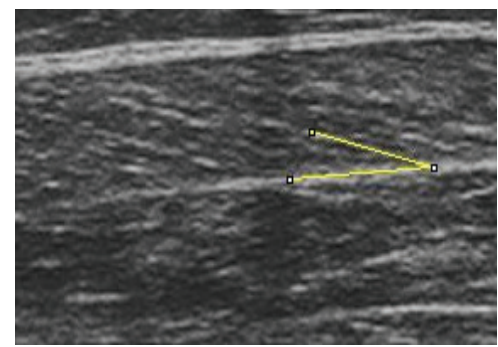

Figura 1. Barra de tarefas do software ImageJ com a ferramente para medição de (F selecionada e a janela com o resultado da medida (A). Detalhe das imagens ultrassônicas das retas traçadas para medição do $C F(B)$ e do AP (C) do GL na região de $30 \%$ proximal do comprimento da perna. 


\section{RESULTADOS}

Os valores das medidas apresentaram distribuição normal e os dados referentes à média, desvio padrão, CV, ICC, ETM de CF e AP dos dois dias encontram-se nas tabelas 1 e 2.

Tabela 1. Valores de média, CV, ICC e ETM do comprimento da fibra (CF) dos músculos GM e GL.

\begin{tabular}{|c|c|c|c|c|c|}
\hline & & média $\pm \mathrm{dp}(\mathrm{mm})$ & CV (\%) & ICC & ETM (mm) \\
\hline \multirow{3}{*}{ GM } & Dia 1 & $38,54(0,88)$ & 2,33 & 0,99 & \\
\hline & Dia 2 & $37,85(0,62)$ & 1,67 & 0,99 & \\
\hline & Dois dias & $38,19(1,61)$ & 4,22 & 0,99 & 2,17 \\
\hline \multirow{3}{*}{ GL } & Dia 1 & $36,20(0,86)$ & 2,41 & 1,0 & \\
\hline & Dia 2 & $36,12(0,84)$ & 2,38 & 0,99 & \\
\hline & Dois dias & $36,16(1,55)$ & 4,44 & 0,98 & 2,25 \\
\hline
\end{tabular}

$\mathrm{CV}=$ coeficiente de variação; ICC = coeficiente de correlação intraclasse; ETM = erro típico da medida; AP = ângulo de penação; GM = gastrocnêmios medial; $\mathrm{GL}=$ gastrocnêmios lateral.

Tabela 2. Valores de média, CV, ICC e ETM do ângulo de penação (AP) dos músculos GM e GL.

\begin{tabular}{|c|c|c|c|c|c|}
\hline & & média $\pm \mathrm{dp}\left({ }^{\circ}\right)$ & CV (\%) & ICC & $\operatorname{ETM}\left(^{\circ}\right)$ \\
\hline \multirow{3}{*}{ GM } & Dia 1 & $22,88(0,90)$ & 3,85 & 0,96 & \\
\hline & Dia 2 & $24,06(0,92)$ & 3,93 & 0,99 & \\
\hline & Dois dias & $23,47(2,64)$ & 11,62 & 0,67 & 3,1 \\
\hline \multirow{3}{*}{ GL } & Dia 1 & $20,58(0,78)$ & 3,8 & 0,98 & \\
\hline & Dia 2 & $20,49(0,91)$ & 4,44 & 0,98 & \\
\hline & Dois dias & $20,54(2,19)$ & 10,69 & 0,83 & 3,7 \\
\hline
\end{tabular}

$\mathrm{CV}=$ coeficiente de variação; ICC = coeficiente de correlação intraclasse; ETM = erro típico da medida; $A P$ = ângulo de penação; GM = gastrocnêmios medial; GL = gastrocnêmios lateral.

A ANOVA com medidas repetidas não identificou diferenças significantes entre as imagens e entre os dias para nenhum dos parâmetros. $\mathrm{O}$ CV das medidas do CF variou de 1,67 a 4,44\% e do AP de 3,8 a 11,62\%.

Em relação ao ICC, com exceção do valor referente aos dois dias do AP do GM $(0,67)$, todos se encontram no intervalo de grande confiabilidade. Os dois valores mais altos de $\operatorname{ETM}\left(3,1^{\circ}\right.$ e $\left.3,7^{\circ}\right)$ correspondem aos dois valores inferiores do ICC $(0,67$ e 0,83 , respectivamente), referente às medidas repetidas interdias, do GM e do GL. Já as medidas de CF de GM

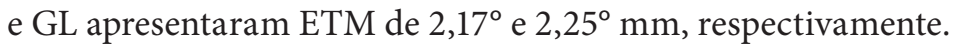

\section{DISCUSSÃO}

Os valores obtidos no presente estudo encontram-se na faixa de pesquisas aplicadas que quantificam o AP e o CF, no entanto, estudos com metodologias similares ${ }^{6,11,21,22}$ não esclareceram informações como o ETM e o ICC, de grande valia na análise das medidas desses parâmetros e para análise da reprodutibilidade e confiabilidade da metodologia empregada. 
As medidas de CF do GM e do GL não apresentaram diferenças significantes entre as imagens e nem entre os dias, sendo 1,67\% o valor mínimo de CV (para o GM, entre as imagens) e o máximo 4,44\% (para o GL, entre os dias). As médias de AP encontram-se na faixa de estudos aplicados, embora apresentem valores de CV maiores, chegando a 11,62 e 10,69\% (inter-dias) para o GM e o GL respectivamente ${ }^{23-27}$. Consequentemente, estes foram os resultados de menor ICC (GM=0,67, GL=0,83) e maior ETM $(\mathrm{GM}=3,1, \mathrm{GL}=3,7)$ o que se justifica, possivelmente, pela dificuldade de realização da medida de AP utilizando software de imagens, pois para tal é necessária a marcação de três pontos na imagem, podendo resultar em um não alinhamento adequado das retas em relação à aponeurose ou à fibra escolhida. Além disso, a escolha da fibra é subjetiva, permitindo escolher em cada imagem uma fibra de melhor visualização. No caso de existirem diferenças entre regiões distintas do músculo, tal escolha pode acrescentar mais erro à medida. É importante frisar ainda que, embora o resultado inferior do ICC tenha sido encontrado nas medidas interdias do AP, o ICC do AP para as medidas do mesmo dia tenha sido foi elevado, ou seja, o erro quantificado não deve ser atribuído unicamente ao procedimento de medição no software, mas à marcação realizada na perna para aquisição das imagens que pode ter sido diferente entre os dias, aumentando as possibilidades de erro. Consideramos esta uma falha metodológica que pode ser reduzida pela elaboração de um molde (em papel) na primeira visita que garanta a posição do transdutor e a aquisição da imagem no mesmo local. Todos os estudos feitos posteriormente pelo grupo já incluíram a utilização do molde sugerido

Um estudo que também utilizou o CV e o ICC para analisar a variabilidade das medidas de arquitetura muscular obtidas através de ultrassonografia é o de Legerlotz et al. ${ }^{15}$. Os autores, com protocolo similar ao do presente estudo, avaliaram a confiabilidade das medidas de CF e AP do gastrocnêmio medial de crianças entre 4-10 anos, adquirindo imagens em duas posições articulares ( $90^{\circ}$ e flexão plantar máxima) e com dois aparelhos de US. Considerando que a amostra era composta por crianças, o estudo apresentou valores de médias absolutas de AP menores que os demais $\left(16,00 \pm 2,40^{\circ}\right)$. Para imagens duplicadas realizadas na mesma sessão, perna e aparelho, os autores encontraram CV e ICC variando, respectivamente, de 4,6 a $6 \%$ e 0,85 a 0,94 para o AP e de 4,9 a $6,0 \%$ e 0,88 a 0,96 para o CF. Esses valores são maiores do que os do presente estudo, provavelmente pela utilização de uma amostra composta por crianças em diferentes fases de desenvolvimento, o que altera os parâmetros de arquitetura muscular (EM, CF e AP). Os autores concluem que o US pode ser um método confiável para determinar a arquitetura muscular do gastrocnêmio em crianças saudáveis, sugerindo que a variabilidade atribuível à metodologia é aceitável, dada a grande variação biológica que pode ser esperada devido a amostra ${ }^{15}$.

Maganaris et al. ${ }^{6}$ testaram a reprodutibilidade de medidas de CF e do AP do tríceps sural e encontraram para o CF resultados de CV similares 
aos do presente estudo ( 4 e 3,8\% para o GM e GL, respectivamente), enquanto para o AP os autores apresentaram valores maiores (8,5\% para o GM e $17,2 \%$ para GL). Ito et al. ${ }^{26}$ encontraram para medições repetidas na mesma imagem de US do AP do tibial anterior um CV de 7\%. Martin et al. ${ }^{7}$ compararam medidas similares em cadáveres e in vivo encontrando valores de CV muito maiores do que os encontrados no presente trabalho $(19,72 \%$ (GM) e $17,7 \%(\mathrm{GL})$ para CF e $25 \%$ (GM) e 28\% (GL) para o AP). No entanto, os autores utilizaram régua e transferidor para medição de $\mathrm{CF}$ e AP nas imagens de US impressas, método que se mostrou menos confiável do que o software de processamento de imagens. Tal fato é ratificado por estudo de Kubo et al. ${ }^{23}$ que relataram CV entre 7,2 e 11,8\%, para o CF do GM cujo método utilizado nas medições consistiu na utilização de um curvímetro para medir o CF e um transferidor para quantificar o AP. Em pesquisa de Kawakami et al. ${ }^{21}$ são reportados valores médios similares de AP do GM com ICC de 0,98, entretanto, com percentuais mais altos de CV $(14,28 \%)$, provavelmente pela grande heterogeneidade da amostra em relação a idade e gênero (homens e mulheres de 3 a 94 anos. Além disso, existem diferenças inerentes à condição de ativação da musculatura estudada (repouso e contração submáxima e máxima) como pode ser visto nos estudos de Blazevich et al. ${ }^{2}$, Martin et al. ${ }^{9}$, Kawakami et al. ${ }^{21}$, Ito et al. ${ }^{26}$, entre outros. Alguns desses autores poderiam argumentar o aumento de dificuldade do processo de medição em músculos contraídos devido à perda parcial de contato do transdutor com a pele, portanto, foi enfatizada a comparação com estudos que utilizaram o mesmo protocolo (tríceps sural relaxado).

É importante destacar que, o transdutor linear de $80 \mathrm{~mm}$ utilizado para a realização das medidas permitiu, na maioria dos casos, o alcance de toda a extensão do comprimento da fibra, não havendo necessidade de extrapolações, garantindo, assim, maior precisão ao método.

Não foi possível encontrar resultados referentes ao ETM de medidas de CF e AP do gastrocnêmio derivados de estudos que utilizaram métodos semelhantes aos aqui apresentados. As medidas de CF de GM e GL apresentaram ETM de 2,17 e 2,25mm, enquanto a medida de AP de GM e GL gerou um ETM de 3,1 e 3,7 $7^{\circ}$, respectivamente, o que indica cautela na utilização para fins de monitoramento de modificações musculares, principalmente, quando são esperadas pequenas alterações.

Estudos que avaliam a plasticidade da arquitetura muscular sob condições diferenciadas de treinamento ou destreino, demonstram que as diferenças encontradas entre as condições superam os erros referentes ao método de quantificação encontrados no presente estudo. As menores diferenças encontradas para os músculos vasto lateral e reto femoral entre as médias dos parâmetros medidos antes e após 5 semanas de três regimes diferentes de treinamento de força foram $3,1^{\circ}(45,58 \%)$ para o AP e $3,74 \mathrm{~cm}$ (22\%) para $\mathrm{CF}^{27}$. Seynnes et al. ${ }^{28}$ relataram aumentos relativos de 7,7 $\pm 1,3 \%$ do AP do quadríceps femoral 3 semanas após o início de um programa de treinamento. Com relação ao mesmo grupamento muscular, Kawakami et al. ${ }^{29}$ relataram aumentos percentuais médios de $29,1 \pm 9,4 \%$ para AP, para 
o músculo tríceps braquial de seis indivíduos submetidos a treinamento de força durante 16 semanas. As diferenças percentuais apresentadas por todos esses estudos superam os maiores coeficientes de variação reportados tanto para o $\mathrm{CF}(4,44 \%)$ quanto para o $\mathrm{AP}(11,62 \%)$ no presente estudo, com exceção do trabalho de Seynnes et al. ${ }^{28}$ no qual as adaptações relatadas estariam na faixa de variabilidade das medições do presente estudo, embora o período de treinamento tenha sido relativamente curto em relação aos outros trabalhos. Ou seja, as adaptações dos parâmetros da arquitetura muscular em tais pesquisas não poderiam ser justificadas por erros de medição ou inerentes ao protocolo de aquisição das imagens, no entanto, esses erros devem ser considerados nas análises, especialmente no que se refere à sua significância prática e clínica.

É importante ressaltar que as técnicas de aquisição de imagens e de quantificação dos parâmetros utilizadas variam entre os estudos, e a maioria deles não relata um estudo de confiabilidade das medidas, dificultando a comparação de resultados e indicando a necessidade de adoção de uma metodologia reprodutível comum às pesquisas aplicadas.

\section{CONCLUSÃO}

Os resultados obtidos nesse estudo demonstraram que a metodologia utilizada para medição de CF e AP realizada em imagens adquiridas através de US apresentou alta confiabilidade, permitindo seu uso com finalidade de monitoramento de modificações provenientes de intervenções como programas de treinamento físico, dietas ou reabilitações. Os CV encontrados são relativamente baixos (1,67 a 4,44\% para CF e 3,8 a 11,62\% para AP) quando utilizados para comparações de estudos com humanos, sem relevância clínica.

\section{REFERÊNCIAS BIBLIOGRÁFICAS}

1. Lieber RL. Skeletal Muscle, Structure, Function and Plasticity: The physiological basis of rehabilitation. Philadelphia: Lippincott Williams \& Wilkins. 2010.

2. Blazevich AJ, Coleman DR, Horne S, Cannavan D. Anatomical predictors of maximum isometric and concentric knee extensor moment. Eur J Appl Physiol 2009;105:869-8.

3. Miyatani M, Kanehisa H, Masamitsu I, Kawakami Y, Fukunaga T. The accuracy of volume using ultrasound muscle thickness measurements in different muscle groups. Eur J Appl Physiol 2004; 91:264-72.

4. Narici MV, Maganaris CN. Plasticity of the muscle-tendon complex with disuse and aging. Exerc Sport Sci Rev 2007;35(3):126-34.

5. Kawakami Y, Fukunaga T. New insights into in vivo human skeletal muscle function. Exerc Sport Sci Rev 2006;34(1):16-21.

6. Maganaris CN, Baltzopoulos V, Sargeant AJ. In vivo measurements of the triceps surae complex architecture in man: implications for muscle function. J. Physiol 1998;512;603-14.

7. Narici MV, Binzonitt T, Hilbrandt E, Falsel J, Terrier F, Carretelli P. In vivo human gastrocnemius architecture with changing joint angle at rest and during graded isometric contraction. J Physiol 1996;496(1):287-97.

8. Rack PMH, Westbury DR. The effects of length and stimulus rate on tension in the isometric cat soleus muscle. J Physiol 1969; 204:443-60. 
9. Martin DC, Medri MK, Chow RS, Oxorn V, Leekam RN, Agur AM, et al. Comparing human skeletal muscle architectural parameters of cadavers with in vivo ultrasonographic measurements. J Anat 2001;199:429-34.

10. Kawakami Y, Abe T, Fukunaga T. Muscle-fiber pennation angles are greater in hypertrophied than in normal muscles. J App Physiol 1993;74(6):2740-4.

11. Chow RS, Medri MK, Martin DC, Leekam RN, Agur AM, McKee NH. Sonographic studies of human soleus and gastrocnemius muscle architecture: gender variability". Eur J Appl Physiol 2000; 82:236-44.

12. Maganaris CN, Baltzopoulos V, Sargeant AJ. In vivo measurements of the triceps surae complex architecture in man: implications for muscle function. J Physiol 1998; 512;603-14.

13. Abe T, Kumagai K, Brechue WF. Fascicle length of leg muscles is greater in sprinters than distance runners. Med Sci Sports Exerc 2000;32(6):1125-9.

14. Muraoka T, Muramatsu T, Fukunaga T, Kanehisa H. Influence of tendon slack on eletromechanical delay in human medial gastrocnemius in vivo. J Appl Physiol 2004;96:540-4.

15. Legerlotz K, Smith HK, Hing WA. Variation and reliability of ultrasonographic quantification of the architecture of the medial gastrocnemius muscle in young children. Clin Physiol Funct Imaging 2010;30:198-205.

16. Ishida Y, Carroll JF, Pollock ML, Graves JE, Leggett SH. Reliability of b-mode ultrasound for the measurement of body fat and muscle thickness. Am J Hum Biol 1992;4:511-20.

17. Ahtiainen JP, Hoffren M, Hulmi JJ, Pietikäinen M, Mero AA, Alevla J, et al. Panoramic ultrasonography is a method to measure changes in skeletal muscle cross-sectional area. Eur J Appl Physiol 2010;108:273-9.

18. Reeves ND, Maganaris CN, Narici MV. Ultrasonographic assessment of human skeletal muscle size. Eur J Appl Physiol 2004;91:116-8.

19. Atkinson G, Nevill AM. Statistical methods for assessing measurements error (reliability) in variables relevant to sport medicine. Sports Med 1998;26(4):217-38.

20. Hopkins WG. Measures of reliability in sports medicine and science. Sports Med 2000;30(1):1-15.

21. Kawakami Y, Ichinose Y, Fukunaga T. Architectural and functional features of human triceps surae muscles during contraction. J Appl Physiol 1998; 85:398-404.

22. Finni T. Structural and function features of human muscle-tendon unit. Scand J Med Sci Sports 2006;16:147-58.

23. Kubo K, Kanehisa H, Fukunaga T. Gender differences in the viscoelastic properties of tendon structures. Eur J Appl Physiol 2003;88:520-6.

24. Kawakami Y, Abe T, Kanehisa H, Fukunaga T. Human skeletal muscle size and architecture: variability and interdependence. Am J Hum Biol 2006;18:845-8.

25. Morse CI, Tolfrey K, Thom JM, Vassilopoulos V, Maganaris CN, Narici MV. Gastrocnemius muscle specific force in boys and men. J Appl Physiol 2008;104: 469-74.

26. Ito M, Kawakami Y, Ichinose Y, Fukashiro S, Fukunaga T. Nonisometric behavior of fascicles during isometric contractions of a human muscle. J Appl Physiol 1998; 85:1230-5.

27. Blazevich AJ, Gill ND, Bronks R, Newton RU. Training-specific Muscle Architecture Adaptation After 5-wk Training in Athletes. Med Sci Sports Exerc 2003; 35:2013-22.

28. Seynnes OR, Boer M, Narici MV. Early Skeletal Muscle Hypertrophy and Architectural Changes in Response to High-intensity Resistance Training J App Physiol 2007;102:368-73.

29. Kawakami Y, Abe T, Kuno SY, Fukunaga T. Training-induced changes in muscle architecture and specific tension. Eur J Appl Physiol 1995;72:37-43.
Endereço para correspondência

Natália Santos da Fonseca Martins. Universidade Federal do Rio de Janeiro. Escola de Educação Física e Desportos. Laboratório de Biomecânica.

Av. Carlos Chagas Filho, 540. Ilha do Fundão.

21941-590 - Rio de Janeiro, RJ. Brasil. E-mail:natalia_sfm@hotmail.com 